\title{
Temperature effects in complementary inverters made with polysilicon source-gated transistors
}

\author{
R. A. Sporea*, Member, IEEE, M. J. Trainor, N. D. Young, J. M. Shannon, S. R. P. Silva
}

Manuscript received December 8, 2014. The work of R. A. Sporea is supported through the Royal Academy of Engineering Academic Research Fellowship Programme.

R. A. Sporea, J. M. Shannon and S. R. P. Silva are with the Advanced Technology Institute, University of Surrey, Guildford, Surrey, GU2 7XH, United Kingdom (*email: r.a.sporea@surrey.ac.uk).

M. J. Trainor was with MiPlaza, Philips Research, High Tech Campus 4, 5656 AE Eindhoven, The Netherlands

N. D. Young is with Philips Research, 101 Cambridge Science Park, Milton Road, Cambridge CB4 OFY, United Kingdom

\begin{abstract}
Through their high gain and low saturation voltage, source-gated transistors (SGTs) have applications in both analog and digital thin-film circuits. In this study we show how we can design SGT-based logic gates which are practically unaffected by temperature variations. We discuss design characteristics which ensure reliable operation in spite of SGT temperature dependence of drain current, and their implications for manufacturability and large signal operation.
\end{abstract}

Key words - Schottky barrier, thin-film transistor, source-gated transistor, complementary logic gate, gain, noise margin, temperature effects, polysilicon

\section{INTRODUCTION}

Source-gated transistors (SGTs) have remarkable properties for thin-film digital circuits [1], as well as for analog applications [2-4]. Their use results in noise-tolerant logic gates, high gain current sources, high noise immunity, and superior power efficiency, and such devices have been demonstrated in a variety of technologies [5-7]. Unlike the rapidly evolving ULSI CMOS, advances in large area thinfilm transistor architecture have been limited, but by basing certain aspects of circuit design on SGTs, major progress can be achieved.

We have previously showed that complementary SGT inverters made using polysilicon device can have exceptionally large gain and superior noise margin due to the low dependence on drain bias of drain current and to lowvoltage saturation [1].

Here, we show the versatility of complementary SGTbased logic, with excellent performance against temperature despite potentially the large temperature dependence (TD) of SGT drain current. We focus on the polysilicon technology for the following reasons: the capability of the SGT device architecture to effectively suppress the kink effect [8-11] which is detrimental to both analog and digital operation; the ability to create complementary circuits (i.e. both $n$ - and $p$ - type devices) with reduced stand-by power and small component count in comparison to unipolar logic [12-17] which is used out of necessity in many emerging technologies. Renewed relevance of polysilicon both through material and technology advances [18-21] and applications [22-25] makes it a rapidly progressing technology.

We support our findings using measurements on polysilicon SGTs and calibrated simulations using the Silvaco Atlas mixed-mode tools [26].

\section{POLYSILICON SOURCE-GATED TRANSISTORS}

\section{A. Fabrication and modeling of source-gated transistors}

Figure 1 shows a schematic cross-section and an optical micrograph of an n-type poly-Si source-gated transistor (SGT).
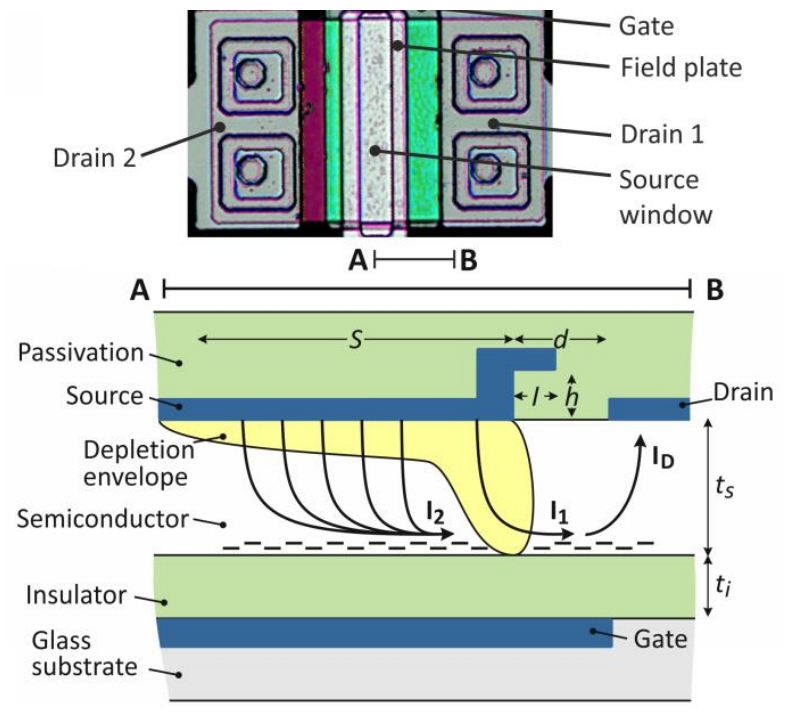

Figure 1. Top - optical micrograph of self-aligned polysilicon SGT $(W=$ $50 \mu \mathrm{m}, d=10 \mu \mathrm{m}, S=10 \mu \mathrm{m}, t_{s}=40 \mathrm{~nm}, t_{i}=300 \mathrm{~nm}$ equivalent oxide thickness, $h=120 \mathrm{~nm}, l=2 \mu \mathrm{m}$ ); bottom - schematic cross-section of the selfligned (gate-drain) SGT with field plate. 
Bottom-gate, staggered electrode devices have been fabricated on glass substrates in a conventional LTPS process using excimer laser crystallization of silicon and self-aligned drain implants by back exposure using the gate as a mask [2] (Fig. 1). After patterning the $\mathrm{Cr}$ gate using photolithography, 200nm of $\mathrm{SiN}_{\mathrm{x}}$ and 200nm of $\mathrm{SiO}_{2}$ were deposited to act as gate insulator $(300 \mathrm{~nm}$ equivalent oxide thickness). $40 \mathrm{~nm}$ hydrogenated amorphous silicon was deposited, baked at $450^{\circ} \mathrm{C}$, and laser crystallized to form the active layer, which was passivated with $120 \mathrm{~nm}$ of $\mathrm{SiO}_{2}$. Top contacts were defined by opening windows in the top insulator and depositing $\mathrm{Cr} / \mathrm{AlTi} / \mathrm{Cr}$. The metal was patterned photolithographically to form overhangs which served as field relief structures. The drain ohmic contact was formed by $\mathrm{n}^{++} \mathrm{P}$ doping. The semiconductor had varying levels of bulk doping for threshold control. Annealing of dopants was performed at $550^{\circ} \mathrm{C}$.

Polysilicon SGTs have desirable properties such as low saturation voltage $\left(V_{S A T I}\right)$ and flat saturated curves (Fig. 2) [2, 4] with suppression of kink effect up to very high drain voltages, tolerance to geometrical variations during fabrication and drain current practically independent on source-drain gap ( $d$ in Fig. 1). Moreover, the flatness of the saturated characteristics can be maintained even for a short $d$ with adequate (and easy to fabricate) field relief structures [27-30].

These devices were the starting point of our circuit simulations with Silvaco Atlas [26]. After fitting the measured curves, the structure was optimized $\left(t_{i}=100 \mathrm{~nm} ; t_{s}\right.$ $=40 \mathrm{~nm}$; field relief plate [30] $h=30 \mathrm{~nm}, l=1 \mu \mathrm{m} ; d=5 \mu \mathrm{m}$; source barrier $\phi_{B O}=0.45 \mathrm{eV}$ ) to achieve low voltage operation with small positive $V_{T}$. The simulations performed were twodimensional and used the cross section of the device (implied width $W=1 \mu \mathrm{m}$ ) divided in a dynamically spaced grid, with coarser spacing in the middle of the s/d electrode gap and at device edges, and finer at the source edge in the $x$ direction and at the semiconductor interfaces in the $y$ direction.

The drain contact was defined as ohmic. The source contact comprised a Schottky barrier to electrons, achieved by enabling the respective flags (barrier, surf.rec) and setting the work function of the source electrode to $4.62 \mathrm{eV}, 0.45 \mathrm{eV}$ higher than the electron affinity of the semiconductor. Barrier lowering was enabled by setting $\alpha=4 \mathrm{~nm}, \beta=1, \gamma=1$, which includes a component proportional to electric field, along with the image force.

Material parameters were chosen to reflect a standard polysilicon process and were set to Atlas defaults. Ambient temperature was kept constant for each simulation run.

The p-type device was defined using the same material parameters and geometry. The nominal barrier of $\phi_{B O}=$ $0.45 \mathrm{eV}$ for holes was generated by choosing the source work function as $0.45 \mathrm{eV}$ lower than the sum of semiconductor electron affinity and its band gap. Transfer characteristics for both n-type and p-type devices are identical to Fig $2 \mathrm{~d}$ in [1].

Circuit simulation was performed using the Atlas mixed- mode capability. The inverter was created using SPICE-like syntax for connecting the components, and defining the $n$ type and p-type devices physically as described above. Transient simulation used the capacitances extracted automatically from the physical simulation.

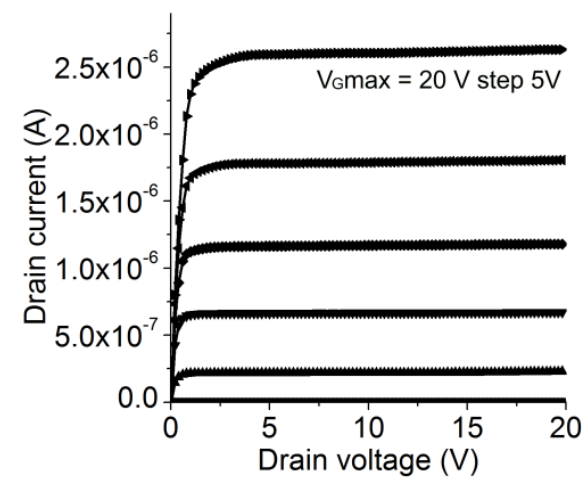

Figure 2. Measured output characteristics of an enhancement-mode polysilicon SGT; $\left(W=50 \mu \mathrm{m}, d=10 \mu \mathrm{m}, S=4 \mu \mathrm{m}, t_{s}=40 \mathrm{~nm}, t_{i}=300 \mathrm{~nm}\right.$ equivalent oxide thickness, $h=120 \mathrm{~nm}, l=2 \mu \mathrm{m}$ ).

\section{B. Temperature effects in SGTS}

SGT drain current can be temperature dependent due to its operation based on the reverse-biased source barrier. Current may increase rapidly with temperature, and along with it, the output conductance $\left(g_{d}=\partial I_{D} / \partial V_{D}\right)$ (Fig. 3), essential for high intrinsic gain $\left(A_{V}=g_{m} / g_{d}\right.$, where $\left.g_{m}=\partial I_{D} / \partial V_{G}\right)$.

By choosing an appropriate source length $(S)$ we can switch from an operating mode which relies to barrier lowering at the edge of the source, with a high TD (short $S$ ), to a mode in which the drain current is dominated by the current from the bulk of the source, with a lower dependence (long $S$ ) [31, 32] (see Fig. 1 for schematic composition of drain current). Fig. 4 illustrates the change in temperaturerelated behavior for extreme values of $S$. The device with a long source has a larger drain current (injected from a larger source area). Significantly, the current originating from the bulk of the source has a lower TD than that of current being injected from the edge of the source electrode and, as a result, the overall temperature dependence of the drain current is lower for longer values of $S$ [31]. This can be explained by the distinct current control processes for $I_{1}$ and $I_{2}$ : reverse saturation current of a Schottky contact for $\mathrm{I}_{1}$; and a resistive effect as $I_{2}$ travels across the semiconductor width, through the accumulated channel and across the depletion region under the edge of the source for $I_{2}$. Fig. 5 shows the gradual transition from one mode of operation (dominated by injection from the edge of the source, high TD) to another (dominated by injection from the bulk of the source, low TD) with increasing $S$. Measured data for two source lengths are presented in the inset. 


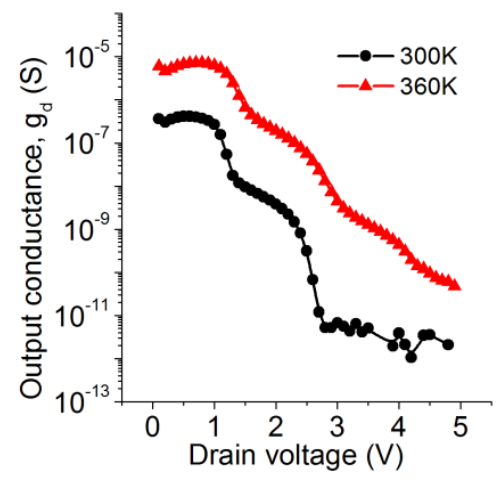

Figure 3. The output conductance, $g_{d}=\partial I_{D} / \partial V_{D}$, of the SGT increases as drain current rises with temperature. $S=5 \mu \mathrm{m}$.

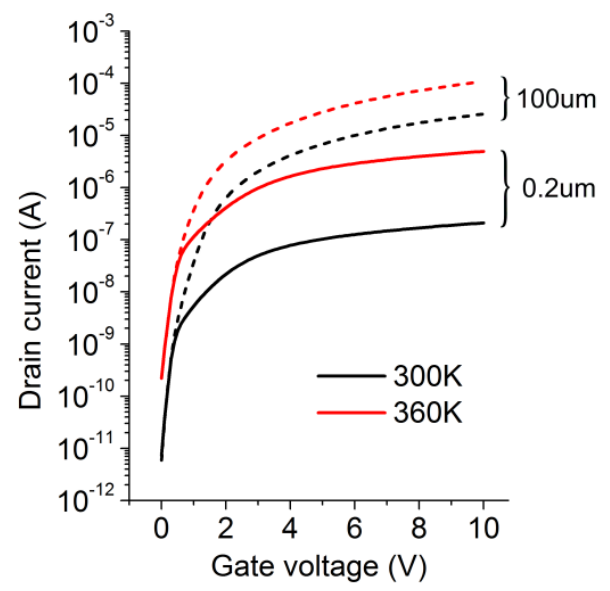

Figure 4. Transfer characteristics of the simulated SGT (n-type) used in the complementary inverter, at two temperatures and two values of $S$. The current increase with temperature is less in the device with a longer source

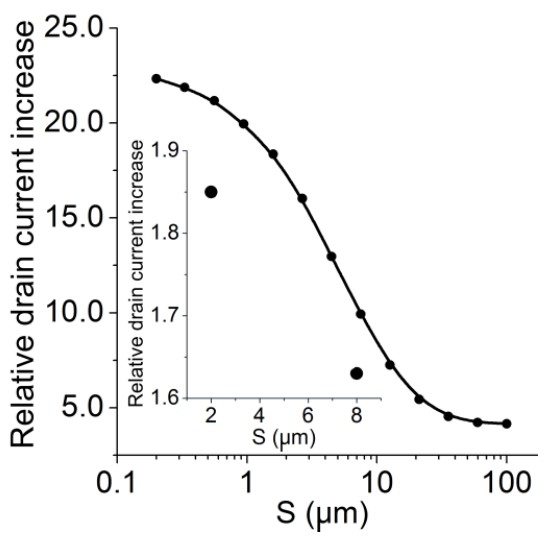

Figure 5. Relative increase of drain current when temperature is changed from $300 \mathrm{~K}$ to $360 \mathrm{~K}$. SGT source length $(S)$ influences drain current sensitivity to temperature: a transition occurs from the first mode of operation (high activation energy, high electric field) to the second mode (low activation energy, low field). Inset: measured dependence of current on temperature and source electrode length on polysilicon transistors.

As $S$ is increased, the saturation voltage does not change (Fig 6a) as it depends on the electrostatics of the depleted semiconductor at the source edge and of the insulator for a given bias condition. This is a useful property which can be exploited in the fabrication of both analog and digital SGT circuits with lower TD when $S$ is used as a design parameter. Fig 6a also shows that for very long $S$ the current saturates with $S$ due to the two-dimensional potential distribution in the source region of the semiconductor [5].

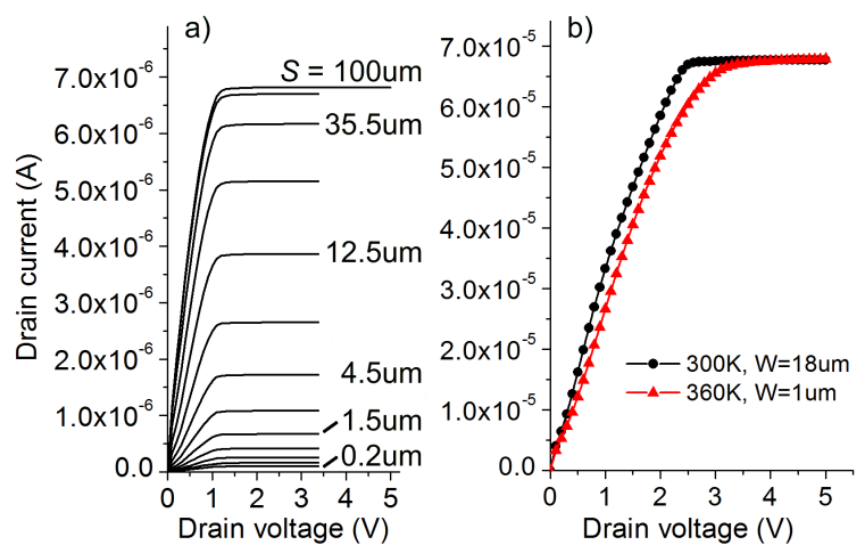

Figure 6. a) Simulated SGT output characteristics for a logarithmic change in $(S)$ from $0.2 \mu \mathrm{m}$ to $100 \mu \mathrm{m}$ at $300 \mathrm{~K}$ and for device width $W=1 \mu \mathrm{m} . V_{G}=$ $5 \mathrm{~V}$. Note that the saturation voltage does not change; b) the output characteristic becomes more rounded as the current increases at higher temperature $(S=4.5 \mu \mathrm{m})$

Likewise, there is only a small saturation voltage shift to higher voltages when temperature increases (Fig. 6b). This is most likely due to the fact that the current density injected at the source is higher as the temperature increases and this larger current follows the envelope imposed by the conductivity of the accumulation channel between source and drain for the respective biasing condition.

\section{SGTS IN DIGITAL CIRCUITS}

\section{A. Complementary SGT inverters}

The electrical characteristics of polysilicon SGTs have been fitted through physical device simulations using Silvaco Atlas [1]. After optimizing the structure for lower voltage operation and positive threshold voltage (see Section II A above), an equivalent p-type device was modelled and complementary inverters (Fig. 7) were constructed using the mixed-mode environment of Atlas in which TCAD and SPICE-type simulations can be concurrently performed.

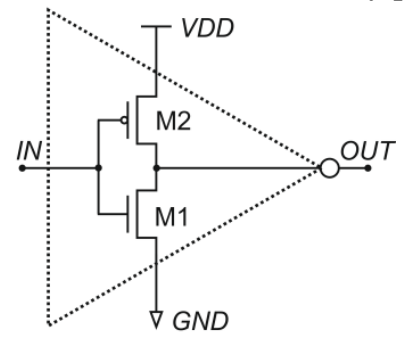

Figure 7. Schematic showing complementary inverter configuration. A.C. simulations were performed using an identical inverter as load at node $O U T$.

The complementary inverters based on polysilicon SGTs show excellent d.c. switching characteristics (Fig. 8) with high noise margin and gain (Fig. 9), and lower power-delay 
product [1] compared to the equivalent FET circuit. These properties recommend SGTs as suitable building blocks for reliable, efficient and easy-to-fabricate large-area digital circuits. Source barrier heights for $p$ - and $n$-type SGTs were chosen so that drain currents would be comparable, and device widths were $W_{p}=2 \mu \mathrm{m}$ and $W_{n}=1 \mu \mathrm{m}$, in keeping with the conventional sizing scheme of silicon-based logic components.

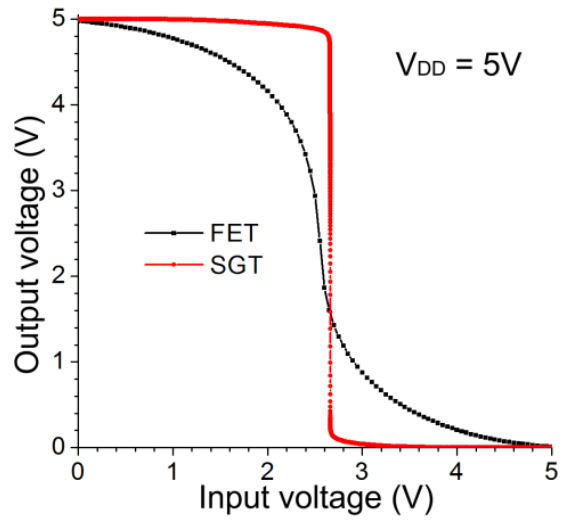

Figure 8. Simulated transfer curves for FET and SGT complementary inverters in polysilicon. Low saturation voltage and flat saturated characteristics allow the SGT circuit to respond closer to ideal than the FET inverter. $S=5 \mu \mathrm{m}$. After [1]

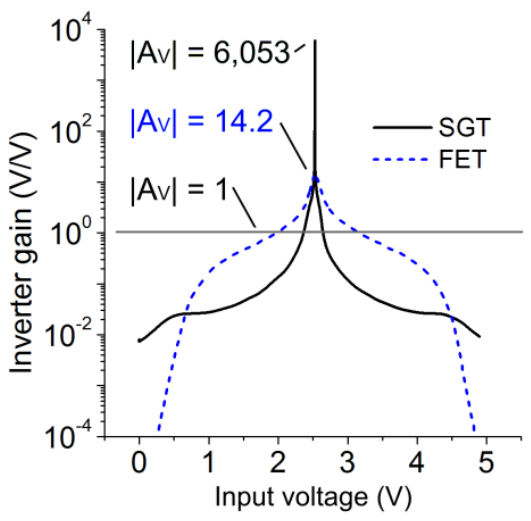

Figure 9. Comparison of complementary inverter gain when made with FETs and SGTs - simulation. $S=5 \mu \mathrm{m}$.

\section{B. SGT inverter performance; temperature and source geometry considerations}

In this study we show that SGT TD is not detrimental to complementary inverter operation. Figures 10 and 11 illustrate the noise margin (NM) and inverter gain for two temperatures and different values of $S$ for complementary SGT inverters.

We see that temperature is having little or no adverse effect on SGT inverters, regardless of $S$. NM (definition incurrent context in the inset of Fig. 10) decreases slightly at high temperature as a result of rounding of output curves around $V_{S A T I}$ (Fig 6b). On the other hand, the inverter gain increases with temperature. We explain this behaviour through the simultaneous increase of $g_{d}$ and $g_{m}$ as the drain current becomes larger at higher temperature. However, the increase in $g_{d}$ is proportionally smaller, due to the saturation mechanism of the SGT and also to field plate functionality, resulting in higher intrinsic gain.

Moderate increases in both metrics are achieved when $S$ increases, particularly in the few-microns range. The SGT circuits significantly outperform the FET equivalent, plotted for comparison in both Fig. 10 and Fig. 11.

Increasing source length benefits the gain and noise margin, but more importantly both NM and inverter gain are not detrimentally affected by increased temperature, regardless of the value of $S$, supporting the conclusion that SGT logic circuits are robust and reliable, despite potentially large TD of device drain current.

Source area, directly linked to $S$, does, however, impact the switching characteristics, through contributions to gate capacitance. The fall time $\left(t_{F}\right)$ for a SGT inverter loaded with an identical circuit is shown in Fig. 12. An optimum value for $S$ exists, since for long $S$ the current does not increase linearly with $S$ (Fig 6a) and for short $S$ the current is impractically small. For this setup, the optimum range is $S=10-30 \mu \mathrm{m}$, which changes only slightly with temperature. This is a useful span of values, considering that conventional polysilicon technologies have design rules in which metal-semiconductor (doped) contact regions are approximately of this size.

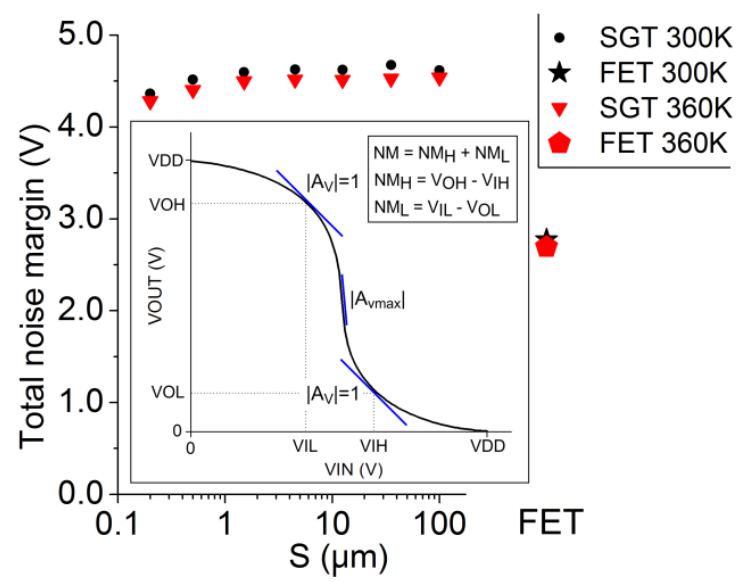

Figure 10. Comparison of FET and SGT (different $S$ values) complementary inverter noise margin. $\mathrm{V}_{\mathrm{D}}=5 \mathrm{~V}$. The degradation with temperature is minimal. Inset - total noise margin calculation, after [1].

Fig. 13 shows that the energy expended per switching event increases linearly with $\mathrm{S}$. The current injected increases with $\mathrm{S}$, but so too does the capacitance which needs to be charged when a similar stage is used as a load. This further supports the preference for a small S.

Fig. 14 illustrates the interrelation between various metrics obtained by changing $S$; NM, gain and fall time all improve as $S$ increases to the optimum. At high temperature, fall time, related to switching speed, is lower, as the current 
increases; gain also improves slightly. Higher gain is achieved simultaneously with faster switching and long $\mathrm{S}$ favors both. Noise margin does not improve significantly for very long $S$. Where switching speed is not important, designing for high gain does not compromise the noise margin. For the fastest switching, a value of $S$ close to the optimum should be used, with minimal penalty in noise margin.

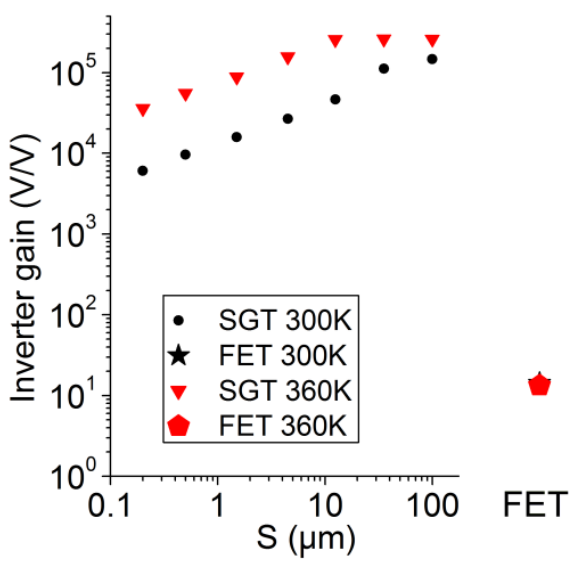

Figure 11. Comparison of FET and SGT (different $S$ values) complementary inverter gain. Gain increases in the SGT inverter due to the rise in drain current (and $g_{m}$ ) with temperature.

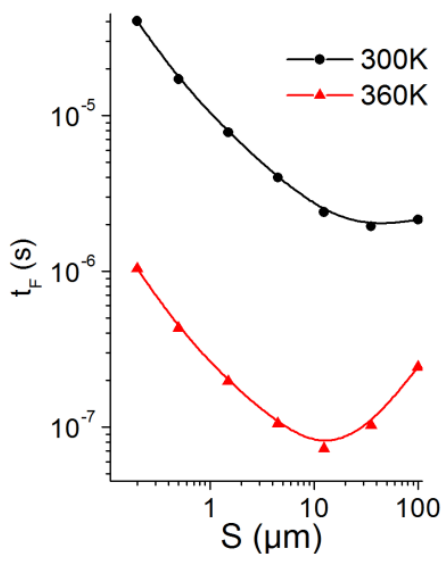

Figure 12. Fall time (to $10 \%$ of $V_{D D}$ ) for the complementary SGT inverter, against $S$ and temperature.

\section{CONCLUSIONS}

Complementary SGT inverters have properties which allow a significant move forward in application performance and robustness for digital large area electronics, with significantly higher gain and noise margin than equivalent FET circuits, as shown in our polysilicon study [1].

Despite the potentially high temperature dependence of SGT drain current, we show that d.c. digital circuit performance does not degrade with temperature. The length of the source electrode, $S$, is a parameter which can be changed in order to lower TD. We show that an optimum value of $S$, taking into account both behavior against temperature and switching speed, exists in the 10-micron range. This is practical from a technological point of view. As the current is controlled at the source, the source-drain gap can be made short without a large penalty in gain, especially for long $S$. As such, choosing the optimum $S$ and minimum source-drain separation will not result in a compact footprint for SGT-based circuit blocks, making this technology easy to implement.

With excellent stability, tolerance to fabrication variability, simplicity of structure, and energy efficiency, SGTs are very promising components for the next generation of robust, high-gain large area thin-film digital and analog circuits, suggesting a potential increase in yield and consistency of performance.

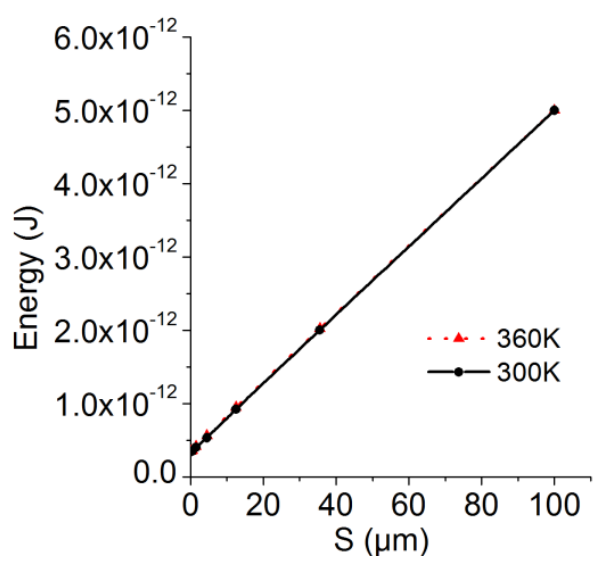

Figure 13. Energy per switching event vs. $S$ for the complementary SGT inverter.

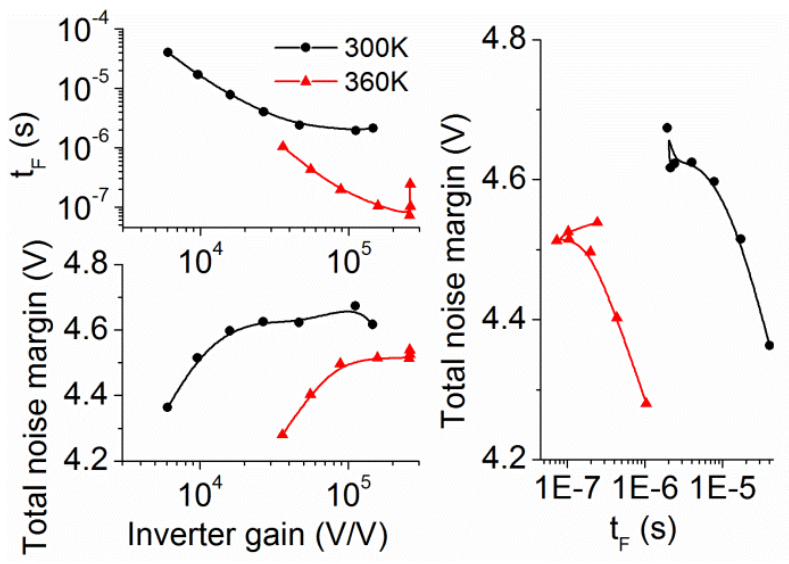

Figure 14. Illustration of the relationship of the performance characteristics of the complementary SGT inverter achieved by changing $S$.

\section{REFERENCES}

[1] R. A. Sporea, M. J. Trainor, N. D. Young, J. M. Shannon, and S. R. P. Silva, "Source-gated transistors for order-of-magnitude performance improvements in thin-film digital circuits," Sci. Rep., vol. 4, 2014. 
[2] R. A. Sporea, M. J. Trainor, N. D. Young, J. M. Shannon, and S. R. P. Silva, "Intrinsic Gain in Self-Aligned Polysilicon Source-Gated Transistors," IEEE Trans. Electron Dev., vol. 57, pp. 2434-2439, 2010.

[3] X. Xiaoli, F. Linrun, H. Shasha, J. Yizheng, and G. Xiaojun, "Solution-Processed Zinc Oxide Thin-Film Transistors With a LowTemperature Polymer Passivation Layer," IEEE Electron Dev. Lett., IEEE, vol. 33, pp. 1420-1422, 2012.

[4] S. D. Brotherton, "Introduction to Thin Film Transistors: Physics and Technology of TFTs", Springer, Cham, 2013.

[5] A. Valletta, L. Mariucci, M. Rapisarda, and G. Fortunato, "Principle of operation and modeling of source-gated transistors," J. Appl. Phys., vol. 114, p. 064501, 2013.

[6] C. Opoku, L. Chen, F. Meyer, and M. Shkunov, "Solution Processable Nanowire Field-Effect Transistors," MRS Proc., vol. 1287, 2011.

[7] A. M. Ma, M. Gupta, F. R. Chowdhury, M. Shen, K. Bothe, K. Shankar, Y. Tsui, and D. W. Barlage, "Zinc oxide thin film transistors with Schottky source barriers," Solid-State Electronics, vol. 76, pp. 104-108, 2012.

[8] G. A. Armstrong, S. D. Brotherton, and J. R. Ayres, "A comparison of the kink effect in polysilicon thin film transistors and silicon on insulator transistors," Solid-State Electronics, vol. 39, pp. 1337-1346, 1996.

[9] M. Hack and A. G. Lewis, "Avalanche-induced effects in polysilicon thin-film transistors," IEEE Electron Dev. Lett., vol. 12, pp. 203-205, 1991.

[10] M. Valdinoci, L. Colalongo, G. Baccarani, G. Fortunato, A. Pecora, and I. Policicchio, "Floating body effects in polysilicon thin-film transistors," IEEE Trans. Electron Dev., vol. 44, pp. 2234-2241, 1997.

[11] A. Valletta, P. Gaucci, L. Mariucci, G. Fortunato, and S. D. Brotherton, "Kink effect in short-channel polycrystalline silicon thinfilm transistors," Appl. Phys. Lett., vol. 85, pp. 3113-3115, 2004.

[12] J.-M. Lee, I.-T. Cho, J.-H. Lee, and H.-I. Kwon, "Full-Swing InGaZnO Thin Film Transistor Inverter with Depletion Load," J. J. of Appl. Phys., vol. 48, p. 100202, 2009.

[13] S. Man Ju, C. Min Hyuk, M. Mativenga, G. Di, K. Deok Yeol, and J. Jin, "A Full-Swing a-IGZO TFT-Based Inverter With a Top-GateBias-Induced Depletion Load," IEEE Electron Dev. Lett., vol. 32, pp. 1089-1091, 2011.

[14] M. Ofuji, K. Abe, H. Shimizu, N. Kaji, R. Hayashi, M. Sano, H. Kumomi, K. Nomura, T. Kamiya, and H. Hosono, "Fast Thin-Film Transistor Circuits Based on Amorphous Oxide Semiconductor," IEEE Electron Dev. Lett., vol. 28, pp. 273-275, 2007.

[15] K. Fukuda, T. Sekitani, T. Yokota, K. Kuribara, T. Huang, T. Sakurai, U. Zschieschang, H. Klauk, M. Ikeda, H. Kuwabara, T. Yamamoto, K. Takimiya, C. Kwang-Ting, and T. Someya, "Organic Pseudo-CMOS Circuits for Low-Voltage Large-Gain High-Speed Operation," IEEE Electron Dev. Lett., vol. 32, pp. 1448-1450, 2011.

[16] H. Tsung-Ching, K. Fukuda, L. Chun-Ming, Y. Yung-Hui, T. Sekitani, T. Someya, and C. Kwang-Ting, "Pseudo-CMOS: A Design Style for Low-Cost and Robust Flexible Electronics," IEEE Trans. Electron Dev., vol. 58, pp. 141-150, 2011.

[17] F. Linrun, T. Wei, Z. Jiaqing, C. Qingyu, J. Chen, and G. Xiaojun, "All-Solution-Processed Low-Voltage Organic Thin-Film Transistor Inverter on Plastic Substrate," IEEE Trans. Electron Dev., vol. 61, pp. 1175-1180, 2014.

[18] T. Shimoda, Y. Matsuki, M. Furusawa, T. Aoki, I. Yudasaka, H. Tanaka, H. Iwasawa, D. Wang, M. Miyasaka, and Y. Takeuchi, "Solution-processed silicon films and transistors," Nature, vol. 440, pp. 783-786, 2006.

[19] R. Ishihara, J. Zhang, M. Trifunovic, M. van der Zwan, H. Takagishi, R. Kawajiri, T. Shimoda, and C. I. M. Beenakker, "Single-Grain Si TFTs Fabricated by Liquid-Si and Long-Pulse Excimer-Laser," ECS Trans., vol. 50, pp. 49-53, March 15, 20132013.

[20] A. Valletta, L. Maiolo, L. Mariucci, A. Pecora, M. Rapisarda, G. Fortunato, and S. D. Brotherton, "Reduction of Short Channel Effects and Hot Carrier Induced Instability in Fully Self-Aligned Gate Overlapped Lightly Doped Drain Polysilicon TFTs," J. Disp. Tech. vol. 8, pp. 18-22, 2012.

[21] Y. Sugawara, T. Oda, T. Saitoh, and K. Komori, "The uniform crystallization process towards the bottom-gated LTPS TFT back- plane technology for large-sized AM-OLED displays by $\mathrm{CW}$ green laser annealing," in AM-FPD Proc., pp. 115-118, 2012.

[22] J. Feng-Renn, F. Yean-Kuen, C. Yen-Ting, C. Tse-Heng, I. L. Cheng, L. Cheng-Wei, and L. Yan-Wei, "Comparative Study of Carbon Monoxide Gas Sensing Mechanism for the LTPS MOS Schottky Diodes With Various Metal Oxides," IEEE Sensors J., vol. 11, pp. 1227-1232, 2011.

[23] T. Kohno, T. Kuranaga, H. Kageyama, M. Ishii, N. Kasai, N. Nakamura, and H. Akimoto, "LTPS AM-OLED Display Consisting of Two-TFTs/One-Capacitor Pixel Circuits for Producing HighUniformity Images," IEEE Trans. Electron Dev., vol. 60, pp. 37803786, 2013.

[24] C. Chih-Yang, L. Chrong-Jung, and K. Ya-Chin, "A New Sensing Scheme for Sensitivity Enhancement of Low-Temperature Polycrystalline Silicon Photodetecors," IEEE Sensors J., vol. 11, pp. 1478-1483, 2011.

[25] L. Maiolo, S. Mirabella, F. Maita, A. Alberti, A. Minotti, V. Strano, A. Pecora, Y. Shacham-Diamand, and G. Fortunato, "Flexible pH sensors based on polysilicon thin film transistors and $\mathrm{ZnO}$ nanowalls," Appl. Phys. Lett., vol. 105, pp. 093501-093501-4, 2014.

[26] "Silvaco Atlas User Manual," 2012.

[27] C. A. Fisher, J. M. Shannon, D. H. Paxman, and J. A. G. Slatter, "The performance of high-voltage field relieved Schottky barrier diodes," Solid-State and Electron Devices, IEE Proceedings I, vol. 132, pp. 257-260, 1985

[28] M. C. Tarplee, V. P. Madangarli, Z. Quinchun, and T. S. Sudarshan, "Design rules for field plate edge termination in SiC Schottky diodes," IEEE Trans. Electron Dev., vol. 48, pp. 2659-2664, 2001.

[29] L. Yong, S. Hongbiao, L. Hai, C. Dunjun, Z. Rong, and Z. Youdou, "Field plate engineering for GaN-based Schottky barrier diodes," IoP J. Semicond., vol. 34, p. 054007, 2013.

[30] R. A. Sporea, M. J. Trainor, N. D. Young, J. M. Shannon, and S. R. P. Silva, "Field Plate Optimization in Low-Power High-Gain SourceGated Transistors," IEEE Trans. Electron Dev., vol. 59, pp. 21802186, 2012.

[31] R. A. Sporea, J. M. Shannon, and S. R. P. Silva, "Temperature Coefficient Engineering in Source-Gated Transistors," Proc. ITC 2014, 23-24 January 2014, Delft, The Netherlands, p. 16, 2014.

[32] L. Mariucci, M. Rapisarda, A. Valletta, S. Calvi, M. Benwadih, R. Coppard, and G. Fortunato, "(Invited) Contact Effects in Organic Thin Film Transistors with Different Device Structures," ECS Trans., vol. 64, pp. 131-142, 2014.

Radu A. Sporea (M'05) received his Engineer's Diploma in 2006 from the Faculty of Electronic Engineering, Information Technology and Telecommunications, 'Politehnica' University of Bucharest and his PhDfrom the University of Surrey in 20101. He holds a Royal Academy of Engineering Research Fellowship at the University of Surrey, working on low power, large area, flexible and printed electronics. In 2014 he was identified as a Rising Star by the Engineering and Physical Sciences Research Council UK. $\mathrm{He}$ is a member of SID and was a Design Engineer for Catalyst Semiconductor.

John M. Shannon was Head of the Display and Large Area Electronics Group at Philips Research, UK (1985 - 1994) before becoming a scientific advisor to Philips Electronics. He is Professor of Semiconductor Devices at the University of Surrey and a Fellow of the Royal Academy of Engineering.

Michael Trainor received his $\mathrm{BSc}$ and $\mathrm{PhD}$ degrees in chemistry from the University of Strathclyde, UK, in 1985 and 1990 respectively. He joined Philips Research in 1989 and since then has worked in the field of polysilicon Thin Film Transistor devices for active matrix displays and novel devices. His main activity has been on technology development and process flow design for LTPS on glass and polymer substrates, prototyping a number of LCD display designs over the years. His most recent work has focused on using LTPS technology to fabricate a PCR (polymerase chain reaction) thermo cycler array with integrated circuitry controlling heaters and temperature sensors. He is currently working with the Technical University of Eindhoven on technology development for photonic ICs. 
Nigel Young received his B.Sc and Ph.D degrees from the University of Leeds, UK, in 1980 and 1984 respectively. Since then he has worked continuously for Philips Research, mainly in the field of LTPS devices and technology for active matrix displays and novel applications. His early work was on device physics and stability, he then worked more on the technology for glass, polymer and steel substrates, demonstrating several LCD and OLED displays based upon this technology. Aside from displays, he has also worked on fingerprint scanners, EEPROMs, lab-on-chip, MEMs and sensors, and has given over 25 invited papers on this broad range of topics. His present focus is on metal-oxide devices, and on new fields of work such as electrochemistry for lifestyle and healthcare.

S. Ravi P. Silva, FREng is the Director of the Advanced Technology Institute (ATI) at the University of Surrey, and has made over 500 presentations at international conferences, has published over 450 journal papers and is the inventor of 30 patents. He has recently concluded one of the most successful Portfolio Partnership awards for $£ 6.68 \mathrm{~m}$ with Engineering and Physical Sciences Research Council (EPSRC) on Integrated Electronics and at present is working closely on 4th generation hybrid solar cells for large area deployment. He is working with the Royal Academy of Engineering and colleagues in India on the large scale deployment of solar technologies in India. He has won the Albert Einstein Silver Medal from UNESCO, the Charles-Vernon-Boys Medal from the Institute of Physics and the J J Thomson Achievement Medal from the Institute of Engineering and Technology. He acts as a technical advisor to Surrey NanoSystems Ltd and to the Sri Lanka Institute of Nanotechnology.
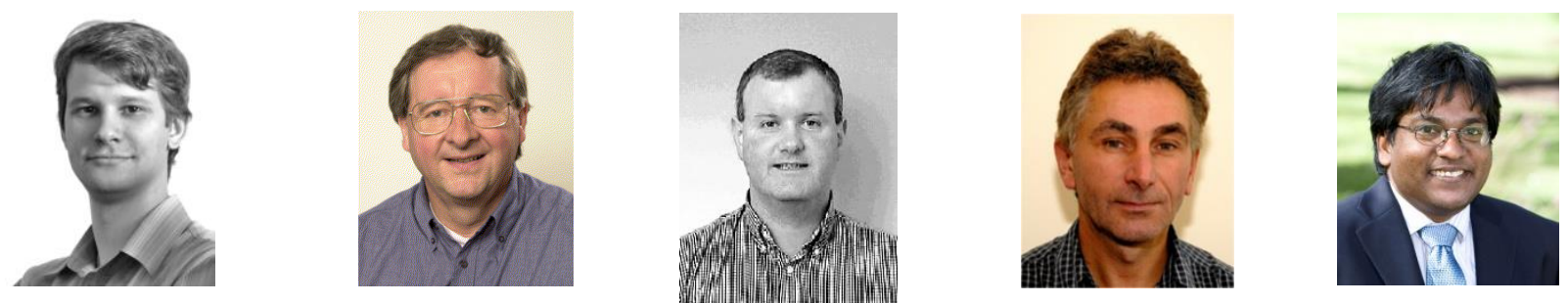